\title{
New separation techniques for output-only modal analysis
}

\author{
Jérôme Antoni ${ }^{\mathrm{a}}$, Luigi Garibaldi ${ }^{\mathrm{b}, *}$, Stefano Marchesiello $^{\mathrm{b}}$ and Menad Sidhamed ${ }^{\mathrm{a}}$ \\ ${ }^{a}$ University of Technology of Compiègne, France \\ ${ }^{\mathrm{b}}$ Politecnico di Torino, Italy
}

Caro Bruno, pensiamo sempre di vedere il tuo sorriso affacciato alla nostra porta, credendo di sentire i tuoi passi in corridoio: ci manchi.

\begin{abstract}
The paper is devoted to the problem of discriminating between operational and natural modes of structures excited by generic inputs. This case often occurs when the system under analysis holds rotating parts and is contemporary excited by ambient noise; in this case the output-only techniques may fail being easily trapped in a misinterpretation of the system eigenvalues. A survey of the methods available in literature is given, together with the explanation of their failures. To solve this problem, two new techniques are introduced and their capabilities are checked with numerical and experimental data from a paper machine.
\end{abstract}

\section{Introduction}

Output-only modal analysis has been widely adopted in recent years as a powerful tool for dynamic linear systems identification. Its success is probably due to the fact that measuring the forcing excitation of a structure is impossible in many cases. Among the algorithms developed and used to extract modal parameters, some have reached a high degree of robustness and reliability, such as the ARMAV and eigensystem realisation type algorithms. Due to their robustness, these algorithms have been extensively adopted in a number of situations, where the white Gaussian input requirement is not fulfilled or, moreover, the input noise is represented by a combination of a coloured noise mixed with pure harmonic components. Quite often, the case occurs when dealing with rotating machinery, where the unbalances play a fundamental role and many harmonics may appear in the output spectrum, so that system eigenfrequencies become hardly masked.

A review of the existing methods is presented along the paper and their performances tested on numerical and real systems. Two new approaches are proposed, based on the presence of pure tones in the output signals; they seem to be robust and give better results if compared with other methods from literature.

\section{Problem statement}

\subsection{Formulation as a test of hypothesis}

A versatile model for the vibration signal of mechanical systems can be interpreted as a mixture of three types of components:

i) periodic components - also referred to as pure tones or harmonics - which usually originate from the forced response of the system to periodic excitation forces,

\footnotetext{
*Corresponding author. E-mai: luigi.garibaldi@polito.it.
} 
ii) structural resonances which originate from the response of the system to transient and random forces,

iii) and background noise which usually accounts for anything else that does not fit in the two previous categories.

In the mathematical language, such a vibration signal can be expressed as

$$
x(t)=\sum_{j=1}^{J} A_{j} e^{2 \Im \pi f_{j} t}+\sum_{k=1}^{K} h_{k}(t) * s_{k}(t)+n(t)
$$

where * denotes convolution, $\Im$ is the complex imaginary, $A_{j}, j=1, \ldots, J$ are the complex coefficients of pure tones positioned at frequencies $f_{i}, h_{k}(t), k=1, \ldots, K$ are the impulse responses of structural resonances excited by some random forces $s_{k}(t)$, and $n(t)$ is a covariance-equivalent process that accounts for the background noise. Model (1) leads to a mixed-spectrum in the Fourier domain, i.e. a mixture of a discrete density -sometimes referred to as a line-spectrum- due to components of type (i) and a continuous density due to components of type (ii) and (iii). Specifically, if $S_{z}(f)$ denotes the power spectral density of signal $z(t)$, then

$$
S_{x}(f)=\sum_{j=1}^{J}\left|A_{j}\right|^{2} \delta\left(f-f_{j}\right)+\sum_{k=1}^{K}\left|H_{k}(f)\right|^{2} S_{s_{k}}(f)+S_{n}(f)
$$

with $\delta(f)$ the Dirac impulse and $H_{k}(f)$ the Fourier transform of $h_{k}(t)$.

The issue we are concerned with in this paper is to state whether, at any given frequency $f$ of the spectrum, a pure tone is present or not. In other words, we wish to test between the two hypotheses:

$$
\left(\begin{array}{l}
H_{0}: \text { : "there is no pure tone at frequency f" } \\
H_{1} \text { : "a pure tone is present at frequency f" }
\end{array}\right.
$$

In theory, this seems not to be a problem at all since a pure tone is materialised by a Dirac impulse in the spectrum and this would have a very different shape than the spectral density of a resonance or of the background noise. This reasoning rapidly turns out to be naive in the real world, because of the uncertainty principle. The uncertainty principle says that a measured signal $x(t)$ cannot have an infinite frequency resolution. A the extreme, if the time record is not long enough, the frequency resolution may be too poor to resolve between a presumed Dirac impulse and a sharp-pointed resonance. This difficulty was early recognised by Bartlett who observed that "given only a finite number of observations and no further information, it is impossible to distinguish strict harmonic components from peaks in the continuous spectrum of arbitrarily small width" [1]. Indeed, distinguishing between the two types of components is made even worse in presence of strong background noise, because the characteristic pedestal of a sharp-pointed resonance then gets completely masked by the noise spectrum, and what emerges above the baseline really looks like the neck of a Dirac. This is illustrated in Fig. 1.

Hence, the actual difficulty in our issue is really to discriminate between pure tones and sharp-pointed resonances buried into strong additive noise. In this respect, the non-trivial part of our test of hypotheses could be reformulated as:

$$
\begin{aligned}
& \text { Discriminate between: } \\
& \left(\begin{array}{l}
H_{0}^{\prime}: \text { :a sharp-pointed resonance is present at frequency } \mathrm{f} \text { " } \\
H_{1}: \text { : "a pure tone is present at frequency f" }
\end{array}\right.
\end{aligned}
$$

\subsection{Requirements}

This paper is largely devoted to comparing different techniques for solving the aforementioned issue. Therefore, it is essential to have a fair basis of comparison on which to draw sound conclusions. We propose to introduce the following requirements which guarantee that any proposed solution has realistic potentials for real world applications.

R1) Independence to the number of tones

The number of pure tones should not be a parameter to be identified. This is a strong requirement when dealing with mechanical systems vibrations where hundreds of tones are commonly encountered.

$\mathrm{R} 2)$ Independence to the nature of noise 


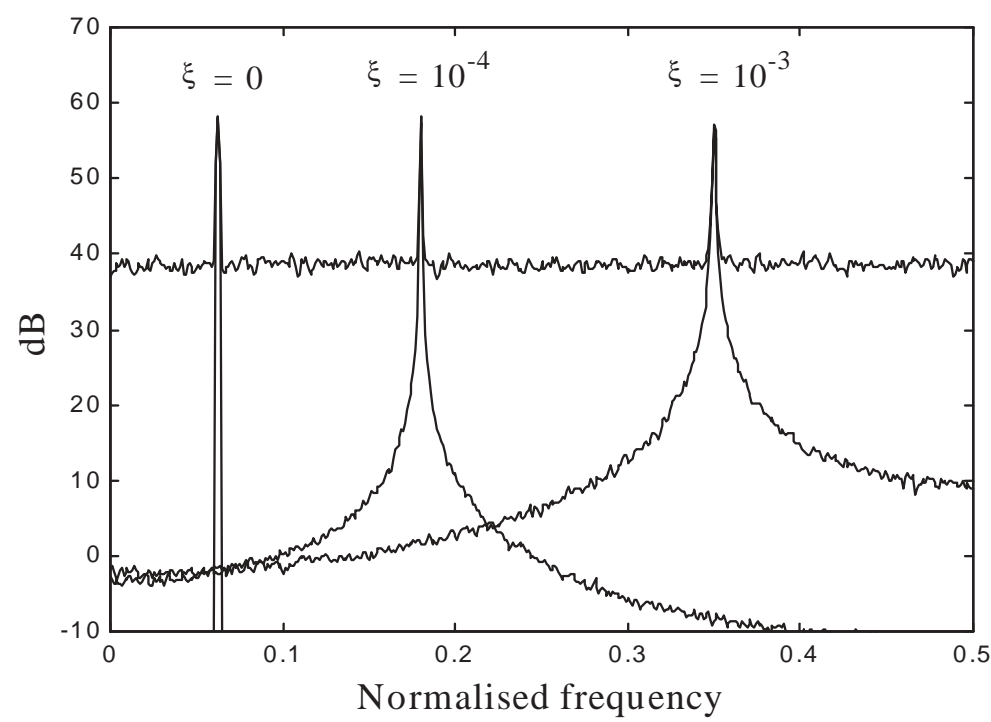

Fig. 1. Example of a mixed-spectrum composed of a pure tone at $f=0.0625$, two sharp-pointed resonances at $f=0.18$ and $f=0.35$ with damping ratios of $10^{-4}$ and $10^{-3}$ respectively, and additive white noise. Global signal-to-noise ratio is $0 \mathrm{~dB}$.

The proposed solution should be able to cope with any type of noise, in terms of colour, probability distribution, and time characteristics. It should not be confined to the usually presumed white Gaussian stationary environment, an assumption that turns out to be unrealistic in most practical instances. Is also included in this requirement the capacity to recognise a sharp-pointed resonance as a coloured "noise" and not as a pure tone.

R3) Robustness to the level of additive noise

The proposed solution should be able to cope with strong additive noise, a situation that again is commonplace when dealing with mechanical systems. When necessary, this quality will be measured by the discrimination power (quality of correctly choosing assumption $H_{0}$ or $H_{1}$ ) versus the signal-to-noise ratio.

R4) Minimum number of parameters to be tuned by the user

The proposed solution should be as transparent as possible to the user and should not rely on too much of his/her intervention.

\section{A brief literature review}

It seems that the issue exposed in this paper, under this point of view, has rarely been addressed in the specialised literature. In this section, we review some of the existing approaches which relate more or less to our concern and could help to make it evolve.

\subsection{Approaches based on periodogram statistics}

These early techniques used the fact that, under the null hypothesis $H_{0}$, high spectral peaks occur with low probability in the periodogram. Any peak higher than a statistical threshold can therefore be associated with a pure tone, given a small probability of error. In the case the noise is white, the statistical threshold is simply computed by averaging the periodogram ordinates. This is the so-called Fisher's g-test which has extensively been used in the past [2]. However, if the noise is coloured, then the statistical threshold becomes a function of frequency and Fisher's test must be adapted. The chief idea proposed so far is to compute the threshold from the underlying continuous spectrum. The difficulty is that the latter is generally unknown and must be obtained from an estimate of the mixed spectrum where all suspicious peaks possibly indicating the presence of pure tones are omitted. But this is exactly what one is looking a technique for! Some approaches have been proposed to force a solution into 
this vicious circle, based on unusual spectral estimates [3,4], but none of them is free of deficiency and, in any case, can deal with sharp-pointed resonances. Therefore requirement R2 is not met. Priestley has suggested an original procedure which apparently alleviates this trouble [5,6], but requirement $\mathrm{R} 4$ precludes its presentation here due to its high complexity.

\subsection{High resolution techniques}

Numerous approaches have been developed in order to bypass the uncertainty principle induced by the Fourier transform. They are referred to as high resolution techniques in the literature, because they can theoretically provide perfect frequency resolution even from finite length records. In essence, most of these approaches rely on the property that the eigendecomposition of the autocorrelation matrix of a mixed signal has its principal eigenspace spanned by the signal (i.e. the set of pure tones) and its orthogonal eigenspace spanned by the noise $[7,8]$. In turn, the dimension of the principal eigenspace also gives the number of pure tones in the signal. However, it is well-known that this theory only works with moderate white noise, but is seriously hampered in the case of coloured noise [9]. Therefore requirement R2 is not met. Alternative approaches have been proposed to cope with coloured noise [10], but the most efficient of them rely on identifying a complete model of the signal, including the noise structure (e.g. by means of an ARMA model) [11]. This is in contradiction with requirement R4, and generally R1.

\subsection{Stochastic subspace identification and stabilisation diagrams}

Hereafter a class of output-only techniques is briefly described, which has gained attention in the last years due to a high level of efficiency and reliability. These techniques are adopted under the assumption that the input on the structure is white. However in many practical situations this assumption is not realistic and if harmonics exist they will be identified as structural resonances, since a non zero damping ratio is estimated.

In case of unknown inputs acting on a structure, a stochastic model can be used to identify the model, i.e., the input terms are implicitly modelled by the noise terms. So, following discrete time stochastic state space formulation is considered [12-14]:

$$
\begin{aligned}
x(k+1) & =A x(k)+w(k) \\
y(k) & =C x(k)+v(k)
\end{aligned}
$$

where $w(k)$ is the process noise vector, due to disturbances and modelling inaccuracies (nonlinearities for example), while $v(k)$ is the measurement noise, due to sensor inaccuracy; $x(t)$ is the state vector and $y(t)$ is the response of the structure. Both $w(k)$ and $v(k)$ are assumed to be zero mean, white vector sequences.

Natural frequencies and damping ratios of the vibrating structure are obtained by performing the eigenvalue decomposition of matrix $A$

$$
A=\Phi \Lambda \Phi^{-1}
$$

while the $r$-th mode shape at the sensor locations is given by the observed parts of the $r$-th system eigenvector

$$
\psi_{r}=C \phi_{r}
$$

The estimation of the matrices $A$ and $C$ in Eq. (3), up to a similarity transformation and using only output measurements $y(k)$, is called the stochastic realisation problem [13] and has been addressed by many researchers in the control and statistics field. In particular, a wide class of methods has recently gained attention for its robustness and efficiency and has been successfully adopted in many circumstances [14-16]. These are called subspace methods because they retrieve system related matrices as subspaces of projected data matrices [12]. In the following, a covariance-driven subspace method is briefly described, namely the Canonical Variate Analysis (CVA).

The first step consists in assembling the block Hankel matrix $H$ and the block Toeplitz matrices $\Re^{+}$and $\Re^{-}[13$, 14]. The Hankel and Toeplitz matrices involve only sampled covariance matrices [17], which are an estimate of the "true" covariance matrices of a stochastic process of infinite length.

The Canonical Variate Analysis requires the computation of the full-rank factorisation of $\Re^{+}$and $\Re^{-}$: 


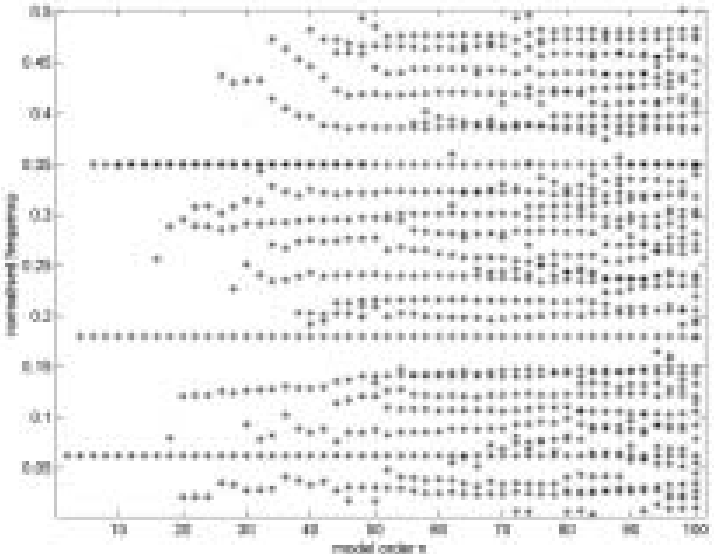

(a)

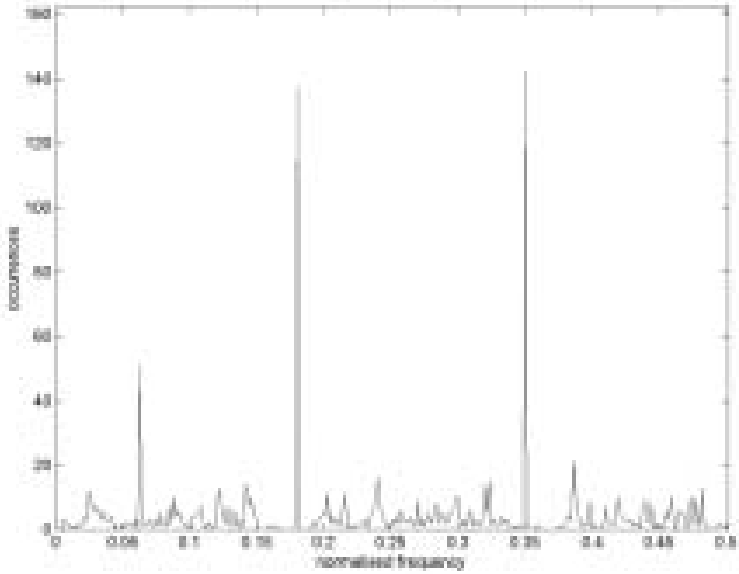

(b)

Fig. 2. CVA on the signal of Fig. 1. (a) Global frequency stabilisation diagram. (b) Occurrence diagram of the extracted frequencies. The two resonances and the pure tone are clearly identified but it is impossible to distinguish between them.

$$
\Re^{+}=L^{+}\left(L^{+}\right)^{T}, \Re^{-}=L^{-}\left(L^{-}\right)^{T}
$$

which gives two weighting matrices for the Hankel matrix $H$. Consequently, a normalised Hankel matrix $H^{*}$ is defined, with the following Singular Value Decomposition:

$$
H^{*}=\left(L^{+}\right)^{-1} H\left[\left(L^{-}\right)^{T}\right]^{-1}=U \Sigma V^{T}
$$

where $\Sigma=\operatorname{diag}\left(\sigma_{1}, \ldots, \sigma_{N}\right), \sigma_{1} \geqslant \sigma_{2} \ldots \sigma_{n} \geqslant 0$.

After some algebraic manipulations [13,17] an estimate is obtained for $A$, whose eigenvectors and eigenvalues give mode shapes, eigenfrequencies and damping ratios for each mode.

The system model order $n$ is generally selected so that $\sigma_{n} \gg \sigma_{n+1}$ (i.e., assuming $\sigma_{n+1}=\ldots=\sigma_{N}=0$ ). However, it is generally not possible to find a strong discontinuity in the decreasing order of the $\sigma$ values so that, in order to select the physical modes, stabilisation diagrams for both eigenfrequencies and damping ratios are needed. The choice of the correct model order $n$ still represents one of the main drawbacks of the CVA method (and of other identification methods) and different criteria are available in the technical literature [17].

In Fig. 2(a) the frequency stabilisation diagram for the signal of Fig. 1 is depicted: if the assumption of white excitation is violated, harmonics are identified as structural modes. The occurrence histogram of the extracted frequencies is shown in Fig. 2(b). It is impossible to distinguish the pure tone from the resonance because in the first case the identified damping ratio is not exactly zero, as shown in Fig. 3.

\subsection{Spectral matrix factorisation}

The idea was initially proposed in [18], and later reintroduced under the new name "Frequency Domain Decomposition" (FDD) in [19]. It can also be seen as an extension of the Complex Mode Indicator Function (CMIF) introduced in [21]. The idea relies on exploiting the interactions between several simultaneous measurements, say

$$
x_{i}(t)=\sum_{j=1}^{J} A_{i j} e^{2 \Im \pi f_{j} t}+\sum_{k=1}^{K} h_{i k}(t) * s_{k}(t)+n_{i}(t)
$$

taken at locations $i=1, \ldots, I$ on the structure. An Hermitian $I \times I$ spectral density matrix $\mathbf{S}_{x}(f)=\left[S_{x_{i} x_{j}}(f)\right]$ is then formed whose diagonal entries contain the auto-spectra of the signals and the out-of-diagonal entries their cross-spectra. It has been claimed that the singular values of the spectral density matrix can indicate whether a spectral peak is due to a pure tone or to a sharp-pointed resonance. The assertion is that a spectral tone shares its power among several singular values whereas a resonance does not $[19,20]$. 


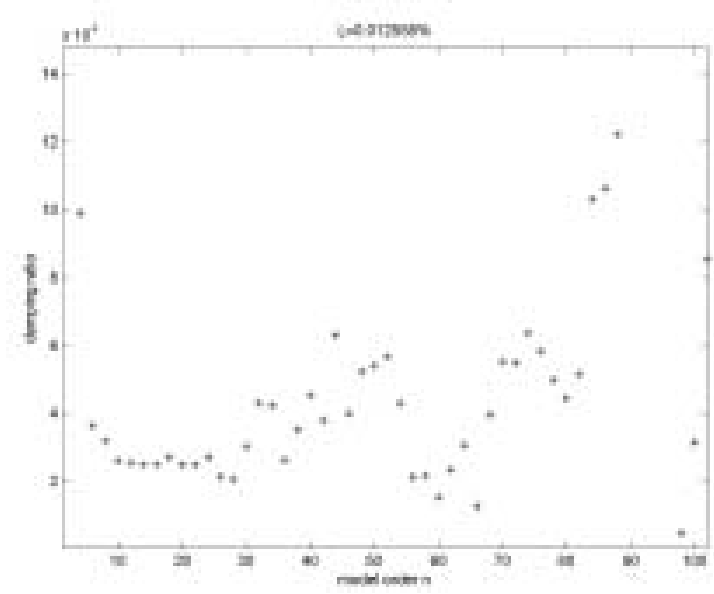

(a)

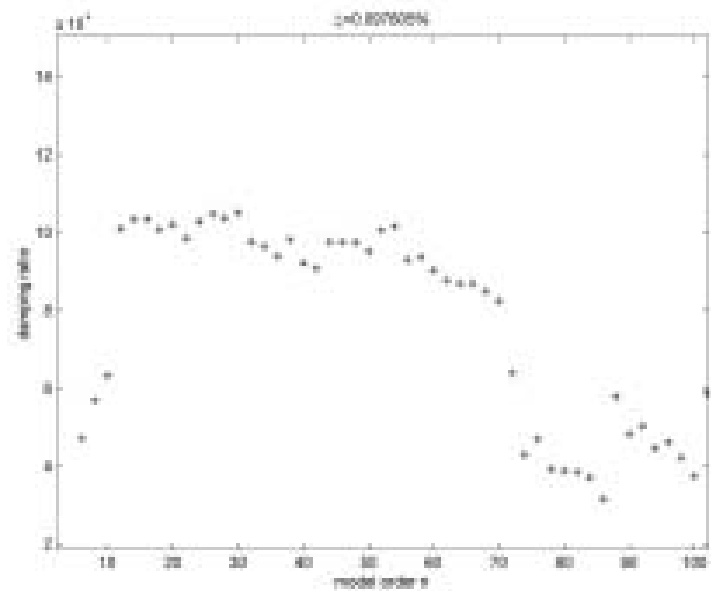

(b)

Fig. 3. CVA on the signal of Fig. 1. (a) Damping stabilisation diagram for the pure tone at $f=0.0625$. (b) Damping stabilisation diagram for the resonance at $f=0.35$.

Unfortunately, despite being occasionally verified on actual data, this assertion does not enjoy the privilege of infallibility and therefore is not a rule. We prove it by means of two counter-examples.

To start with, let us consider the full-expression of the spectral density matrix

$$
\mathbf{S}_{x}(f)=\mathbf{A D}(f) \mathbf{A}^{*}+\mathbf{H}(f) \mathbf{S}_{s}(f) \mathbf{H}(f)^{*}+\mathbf{S}_{n}(f)
$$

where $\mathbf{A}=\left[A_{i j}\right]$ is the matrix of the complex coefficients of the tones, $\mathbf{D}(f)=\left[\delta\left(f-f_{j}\right) \cdot \delta_{i j}\right]$ is a diagonal matrix ${ }^{1}$ containing Dirac impulses, $\mathbf{H}(f)=\left[H_{i j}(f)\right]$ is the transfer matrix containing possible resonances and the exponent $*$ stands for the transpose conjugate operator.

a) First counter-example

For frequency $f=f_{j}$ where a pure tone occurs (assumption $H_{1}$ ),

$$
\mathbf{S}_{x}\left(f_{j}\right)=\mathbf{a}_{j} \mathbf{a}_{j}^{*} \delta(0)+\mathbf{H}\left(f_{j}\right) \mathbf{S}_{s}\left(f_{j}\right) \mathbf{H}\left(f_{j}\right)^{*}+\mathbf{S}_{n}\left(f_{j}\right)
$$

where $\mathbf{a}_{j}$ stands for the $\mathrm{j}^{\text {th }}$ column of matrix A. In theory, since $\delta(0)=\infty$,

$$
\mathbf{S}_{x}\left(f_{j}\right)=\mathbf{a}_{j} \mathbf{a}_{j}^{*} \delta(0)
$$

which is a rank-one matrix. This means that the power of the pure tone at $f=f_{j}$ is entirely carried by the unique nonzero singular value of the spectral density matrix, a property that violates the aforementioned assertion. Of course, due to the use of finite length records in spectral estimates, the estimated power of the signals at frequency $f=f_{j}$ never goes to infinity. But Eq. (11) will still hold approximately whenever the signal-to-noise ratio of a pure tone is high.

b) Second counter-example

For frequencies $f \neq f_{j}, j=1, \ldots, J$ where only noise is present (assumption $H_{0}$ ),

$$
\mathbf{S}_{x}(f)=\mathbf{H}(f) \mathbf{S}_{s}(f) \mathbf{H}(f)^{*}+\mathbf{S}_{n}(f)
$$

In general, the spectral density matrix of Eq. (12) will share its power among several singular values, except in the following special case:

i) $\mathbf{S}_{s}(f)$ is a rank-one matrix and

ii) $\mathbf{S}_{s}(f)=\sigma_{n}^{2}(f) \cdot \mathbf{I}$ i.e. is spherical.

\footnotetext{
${ }^{1} \delta_{i j}$ stands for the Kronecker symbol which equals 1 when $i=j$ and 0 otherwise.
} 


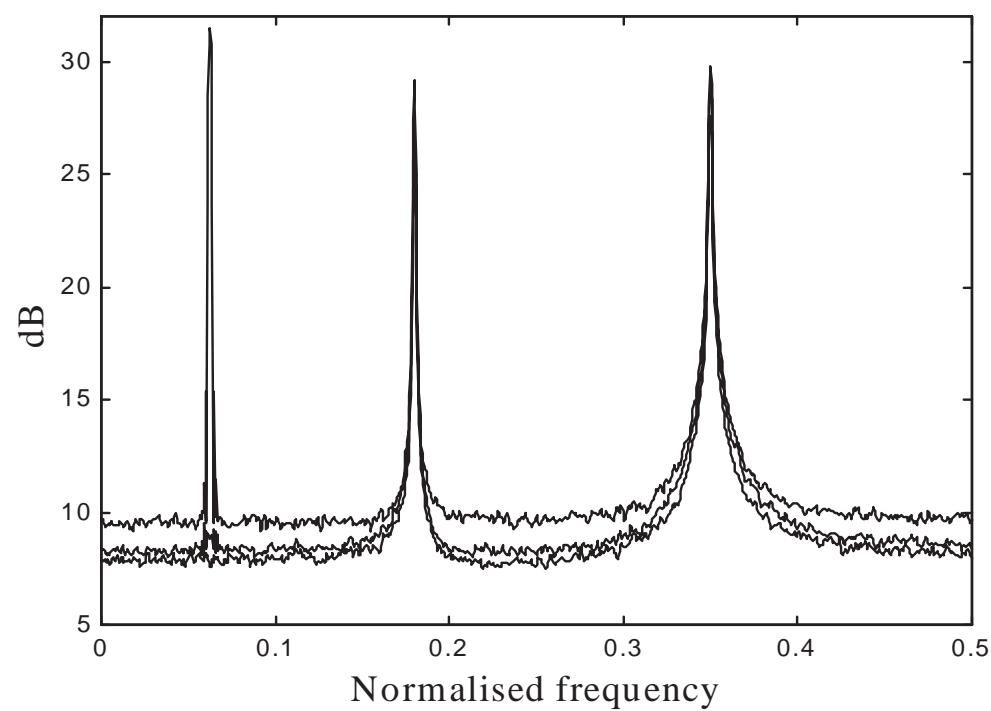

Fig. 4. A typical example where the FDD technique is taken at fault. Three output signals were simulated by exciting a linear system with three independent white forces. Resonances are as in Fig. 1. Independent additive noises were added on the outputs such as to produce a signal-to-noise ratio of $20 \mathrm{~dB}$.

Property (i) means that only one excitation force impinges on the system, and property (ii) that the noises are spatially uncorrelated and of equal power (or negligible), respectively. In all other cases not satisfying (i) and (ii), the aforementioned assertion is again violated. This demonstrates that it is straightforward to construct examples where the "spectral matrix factorisation" approach does not work. Figure 4 illustrates such an example.

\subsection{Approach based on amplitude distributions}

It has been suggested that signals stemming from pure tones and resonances can be differentiated from their amplitude distributions. Indeed, the amplitude distribution of a sinusoid has a characteristic basin-like shape (i.e. bimodal with two maxima at its extremities and a minimum at its center) that is very different from the bell-shaped distribution of a resonance, which actually can be shown to be asymptotically Gaussian as a result of the central limit theorem. A successful application of this idea is presented in [20]. However, what is really tested there is whether a frequency component is a pure tone or not, but not whether a pure tone is present or not. This subtle difference occults the fact that a pure tone may possibly be embedded in additive noise and, actually, no results were given on how this latter situation may affect its theoretical amplitude distribution. Another shortcoming of this approach is that it necessitates demodulating the signals at each frequency of interest, which rapidly becomes cumbersome when many spectral peaks are to be tested. Therefore, this approach does not satisfy requirement R4 and requirement R3 remains to be proved.

\subsection{Approach based on "families of spectra"}

This is probably the most relevant solution to the present issue that has been proposed so far. It exploits the fact that spectral estimates of a pure tone have amplitudes that grow without bound as the duration of the record increases (it approaches the theoretical Dirac impulse), whereas spectral estimates of a random noise stabilise around a constant (the expected value of the noise power). Therefore, superposing on the same plot several spectral estimates corresponding to different record lengths readily indicates where the pure tones are, if any.

The original work of [22] and [23] demonstrated this mechanism for the Minimum Variance estimate. Actually, the Minimum Variance estimate does not show clear advantage in the case of long data records as is commonplace in vibration analysis, and requires tremendous computational efforts. Therefore we advocate applying the same idea to classical spectral estimates based on the smoothed or averaged periodogram. Into addition, we propose a 


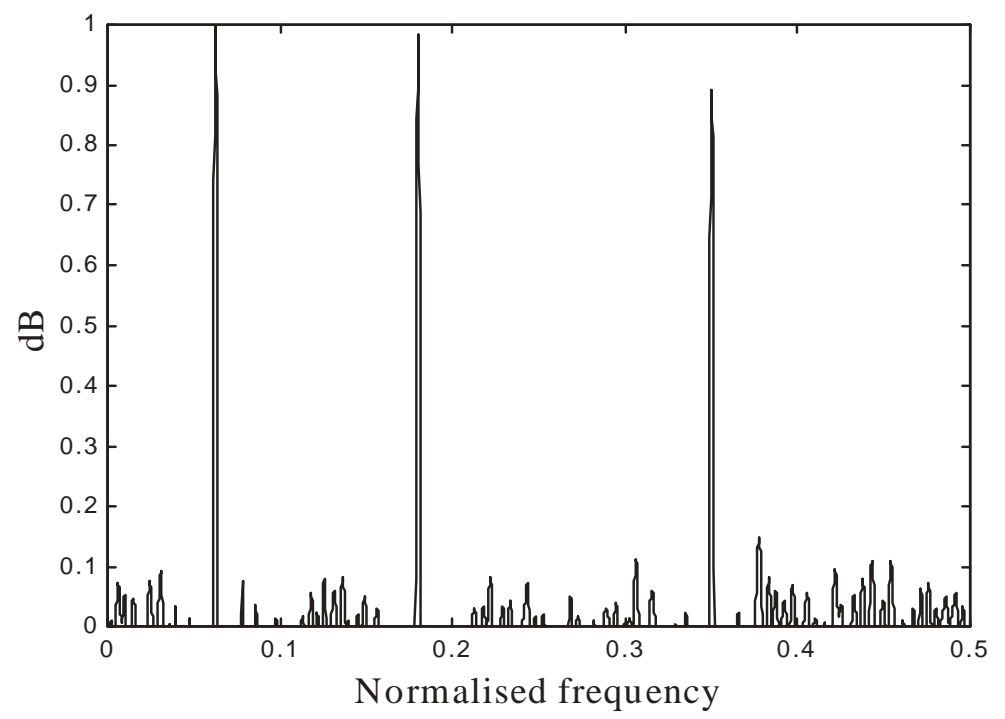

Fig. 5. Estimated indicator $R_{q}(f)$ on the signal of Fig. 1, with $q=2$. Note that the pure tone at $f=0.0625$ is correctly indicated, but the two resonances at $f=0.18$ and $f=0.35$ are incorrectly recognised as tones. Virtually similar results have been obtained using the Minimum Variance estimate, however at the price of much more severe computational efforts.

refined indicator that tries to give a binary (0 or 1) answer. Specifically, if $\hat{S}_{x}^{(q N)}(f)$ and $\hat{S}_{x}^{(N)}(f)$ are the averaged periodograms computed on $q N(q>1)$ and $N$ points, respectively,

$$
R_{q}(f)=\frac{1}{q-1}\left(\frac{\hat{S}_{x}^{(q N)}(f)}{\hat{S}_{x}^{(N)}(f)}-1\right)=\left\{\begin{array}{ll}
1 & \text { under } H_{1} \\
0 & \text { under } H_{0}
\end{array} \quad q>1\right.
$$

But again, there is a subtle difference between this ideal binary conclusion and what is obtain in practice, since a pure tone is unlikely to occur alone, but rather in conjunction with additive noise -this important point was occulted in the aforementioned references. This means that the presence of a pure tone is indicated by a value of $R_{q}(f)$ somewhere between 0 and 1 .

From now on, it seems that the "family-of-spectra" approach fulfils all requirements R1 to R4. However simulations have shown that it hardly meets requirement $\mathrm{R} 2$ when it is faced with very sharp-pointed resonances, as illustrated in Fig. 5.

\subsection{Discussion}

The above review has shown that none of the existing approaches are fully satisfactory with respect to the assigned requirements R1-R4. Indeed, most approaches can hardly discriminate between a tone and a resonance. Despite these shortcomings, the periodogram and the refined "family-of-spectra" approaches should however be acknowledged for trying to propose a binary ( 0 or 1$)$ answer as to whether a frequency component is a pure tone or not.

As a conclusion, there is a need for developing new techniques that evidence better efficiency than the existing ones. In the next two sections, we introduce two new solutions based on original but different approaches. The first one takes advantage of the fact that pure tones have deterministic envelopes. The second one exploits the difference that exists between the correlation lengths of tones and random noises.

\section{A solution based on envelope statistics}

From the statistical point of view, a pure tone is a deterministic signal, i.e. it can be perfectly predicted from a finite number of its past values. Therefore, it has a non-zero expected value which equals itself (almost surely), and 
(a)

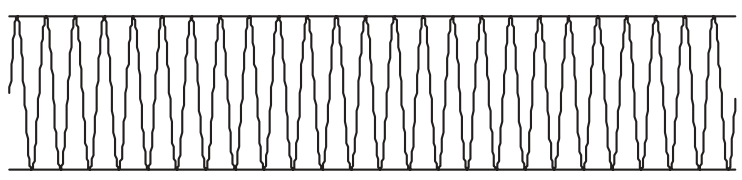

(b)

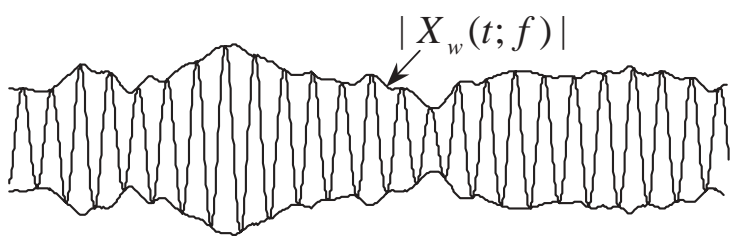

Fig. 6. The envelope indicator is based on the property that the envelope of a pure tone is constant (a), whereas that of a random noise is random (b).

its variance is identically zero. On the other hand, a random noise, by definition, has a non-zero variance and this is independently of its spectral density and probability density function. These observations are relative to ensemble statistics, but they also hold for certain time statistics provided the signals are ergodic (which simply means that time statistics converge to deterministic values, whatever these values).

We demonstrate here how the above observations apply to the envelope of frequency components. Specifically, let $X_{w}(t, f)$ be the discrete-time Fourier transform of signal $x(t)$ along a data segment of length $N$, anchored at time $t$, and tapered by a data window $w(t)$. The quantity $\left|X_{w}(t, f)\right|$ is usually viewed as a function of frequency, e.g. a collection of periodograms indexed by time $t$. It can also be viewed as a function of time, the interpretation being that of the envelope of the frequency component at frequency $f$. If $x(t)=A_{j} e^{2 \Im \pi f_{j} t}$ is a pure tone, then it is an easy matter to check that $\left|X_{w}(t, f)\right|=\left|A_{j}\right| \cdot|W(0)|, f=f_{j}$ with $W(f)$ the discrete-time Fourier transform of the data window $w(t)$. In this case, the envelope is a constant value over $t$. Therefore, its temporal variance is

$$
\operatorname{Var}_{t}\left\{\left|X_{w}(t, f)\right|\right\}=\left\langle\left|X_{w}(t, f)\right|^{2}\right\rangle-\left\langle\left|X_{w}(t, f)\right|\right\rangle^{2}=0
$$

where $\langle\cdot\rangle$ denotes the time average operator. On the other hand, if $x(t)=n(t)$ is a purely random noise (null hypothesis), then the envelope $\left|X_{w}(t, f)\right|$ is itself a random quantity and there will exist a non-empty set of frequencies $f$ such that $\operatorname{Var}_{t}\left\{\left|X_{w}(t, f)\right|\right\}>0$. This idea is illustrated in Fig. 6. The exact value of the temporal variance depends on the time-distribution of the envelope, a quantity that, in general, is unknown. However, it can be shown under mild condition that it is lower bounded by the variance obtained from invoking the Central Limit theorem, i.e. by assuming that $X_{w}(t, f)$ is distributed according to a complex normal law. Since the modulus of a complex normal variable is Rayleigh distributed, it comes that ${ }^{2}$

$$
\frac{\operatorname{Var}_{t}\left\{\left|X_{w}(t, f)\right|\right\}}{\left\langle\left|X_{w}(t, f)\right|^{2}\right\rangle} \geqslant 4-\pi>0, \quad f \neq 0 \bmod (1 / 2)
$$

Results of Eqs (14) and (15) suggest the following binary indicator:

$$
I(f)=\frac{1}{\pi-4}\left(\pi \frac{\left\langle\left|X_{w}(t, f)\right|^{2}\right\rangle}{\left\langle\left|X_{w}(t, f)\right|\right\rangle^{2}}-4\right), f \neq 0 \bmod (1 / 2)
$$

which ideally takes the values:

$$
\left\{\begin{array}{l}
I(f) \leqslant 0, \text { under } H_{0} \\
I(f)=1, \text { under } H_{1}
\end{array}\right.
$$

Note that this result is independent of the nature of the noise, be it normally distributed or not, be it stationary or not, and be it large-band or not. This is why it is expected to work well, in particular when it will come to distinguish between pure tones and sharp-pointed resonances.

\footnotetext{
${ }^{2}$ The symbol mod stands for modulo. It appears here as a result of taking the discrete Fourier transform of sampled data, which has a real value at every frequency $f=k / 2, k \in Z$.
} 


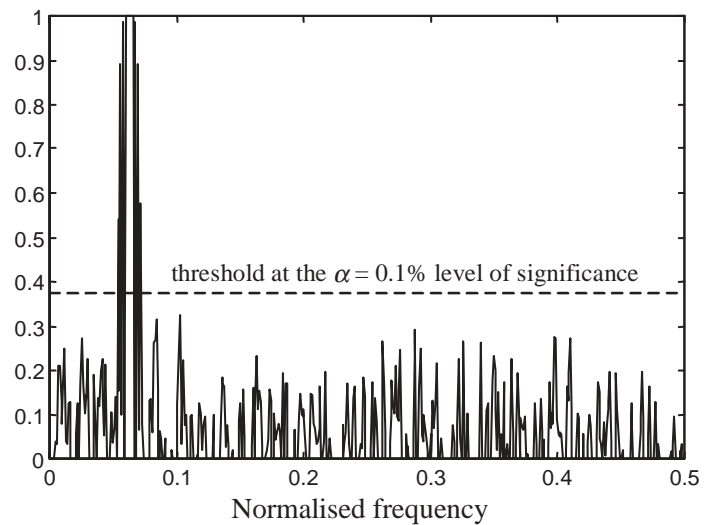

(a)

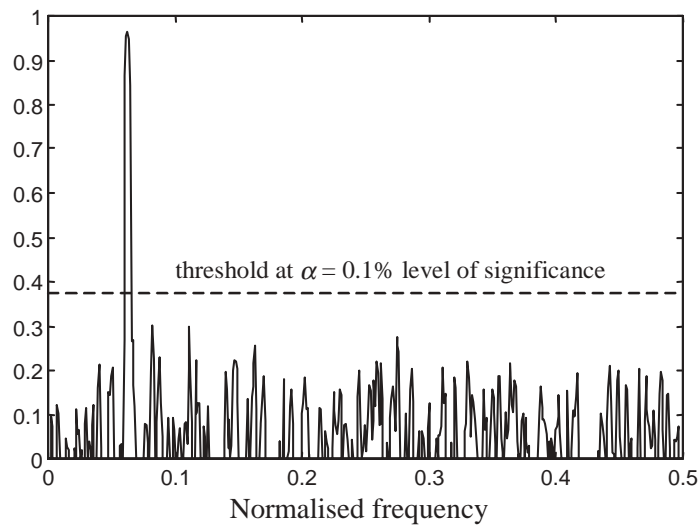

(b)

Fig. 7. Estimated indicator $I(f)$ on the signal of Fig. 1. (a) Signal-to-noise ratio $=\infty$. (b) Signal-to-noise ratio $=0 \mathrm{~dB}$. The two resonances are correctly recognised as not being tones. Note the high level of estimation noise.

The proposed indicator can easily be estimated from a single data record, for instance by using the Welch's method. The so-defined estimator is asymptotically unbiased and consistent, independently of the choice of the data window. Into addition, it can be combined with a statistical threshold which indicates which spectral peaks are statistically believed to be pure tones, as illustrated in Fig. 7.

\section{A solution based on correlation time}

A fundamental property which has not been exploited so far, is that a pure tone has a periodic autocorrelation function, whereas a random noise has an autocorrelation function that ultimately dies to zero. Specifically, if $R_{x}(\tau)=\langle x(t+\tau) x(t)\rangle$ denotes the (time-averaged) autocorrelation function of signal $x(t)$, then $R_{x}(\tau)$ contains a periodic waveform at frequency $f_{j}$ if $x(t)=A_{j} e^{2 \Im \pi f_{j} t}$, and $R_{x}(\tau) \rightarrow 0$ as $|\tau| \rightarrow \infty$ if $x(t)=n(t)$. This idea is illustrated in Fig. 8. More formally, let us define the correlation time of signal $x(t)$ as that time-lag $\tau_{C}$ such that

$$
\left|R_{x}(\tau)\right|<\varepsilon \cdot R_{x}(0), \forall|\tau| \geqslant \tau_{C}
$$

where $0<\varepsilon<<1$ is set arbitrarily small by the user ( $\tau_{C}$ may be seen as the effective time it takes for $R_{x}(\tau)$ to expand fully until it dies to zero). Hence, clearly $\tau_{C}=\infty$ under $H_{1}$ and $\tau_{C}<\infty$ under $H_{0}$.

The above discussion suggested that the behaviour of $R_{x}(\tau)$ at large values of $\tau$ can be used as a discriminating tool for the issue at hand. In fact, Priestley was the first to propose a solution based on this property to detect the presence of harmonics in a time series [5,6]. We propose here a solution that is believed to be technically simpler. It is based on the work presented in reference [24,25]. The idea may be summarised as follows.

Consider two segments of data $x(t+n)$ and $x(t+n+N+\Delta), n=0, \ldots, N-1$ where the delay $\Delta$ is non-negative so that the segments do not overlap. Segment $x(t+n+N+\Delta)$ may be seen as the future version of segment $x(t+n)$ and, as such, it is legitimate to wonder if it can be linearly predicted from the latter. To answer this problem, it is more convenient to switch to the frequency domain, where the data segments are replaced by their discrete-time Fourier transforms $X_{w}(t, f)$ and $X_{w}(t+N+\Delta, f)$. It is well known then that the desired optimal prediction filter has complex gain given by

$$
G^{\Delta}(f)=\frac{\left\langle X_{w}(t+N+\Delta, f) X_{w}^{*}(t, f)\right\rangle}{\left\langle\left|X_{w}(t, f)\right|^{2}\right\rangle}
$$

On the one hand, if $x(t)=A_{j} e^{w \Im \pi f_{j} t}$, then any future segment of data is perfectly predictable, whatever the value of $\Delta$, because the correlation time $\tau_{C}$ of the signal is infinite [23]. On the other hand, if $x(t)=n(t)$, then the prediction is less and less accurate as $\Delta$ becomes large - when $\Delta$ has become greater than the correlation time $\tau_{C}$, 
(a)

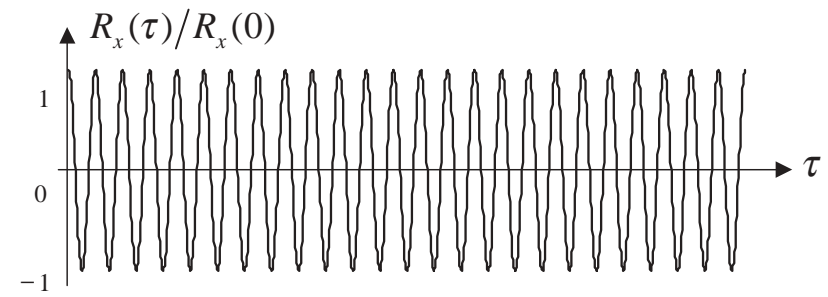

(b)

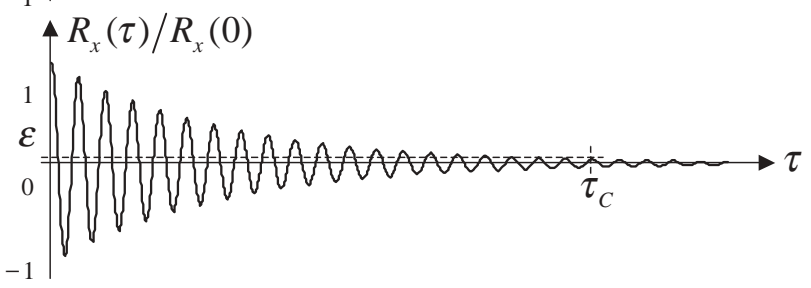

Fig. 8. The correlation time indicator is based on the property that the autocorrelation function of a pure tone has an infinite duration (a), whereas that of a random noise is transient (b).

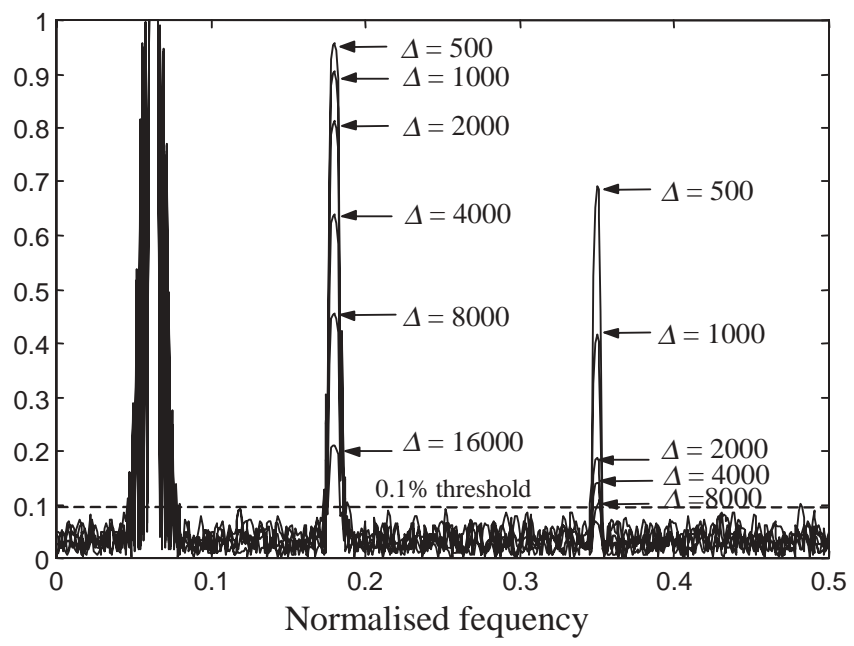

Fig. 9. Estimated indicator $\left|G^{\Delta}(f)\right|$ on the signal of Fig. 1. Signal-to-noise ratio $=\infty$. Results are superposed for different values of the delay $\Delta$. The two resonances are revealed by the convergence to zero of the indicator, with increasing $\Delta$.

the best predicted value is virtually zero. Therefore,

$$
\left\{\begin{array}{l}
\left|G^{\Delta}(f)\right|=1, \quad \forall \Delta \text { under } H_{1} \\
\left|G^{\Delta}(f)\right|=O(\varepsilon), \Delta \geqslant \tau_{C} \text { under } H_{0}
\end{array}\right.
$$

Result Eq. (20) naturally suggests using $\left|G^{\Delta}(f)\right|$ as an indicator.

The indicator $\left|G^{\Delta}(f)\right|$ is easily estimated from a single data record, for instance by using the Welch's method. Again, a statistical threshold can be placed on its estimate, so that the procedure perfectly fulfils requirements R1, R3 and R4 of section III. The only possible difficulty with this strategy is how to set the delay $\Delta$, since its requested minimum value depends on the unknown correlation time $\tau_{C}$, which can be extremely large in case of a sharp resonance. We have found that the best solution is to plot the indicator for increasing values of $\Delta$, say $\Delta_{k}, k=0,1,2, \ldots, \Delta_{k+1}>\Delta_{k}$. This is not really computationally demanding when the Fast Fourier Transform algorithm is used to estimate Eq. (19). Then, those spectral peaks which tends to zero with increasing $\Delta_{k}$ can unambiguously be associated with random components. Figures 9 and 10 illustrate this fact. 


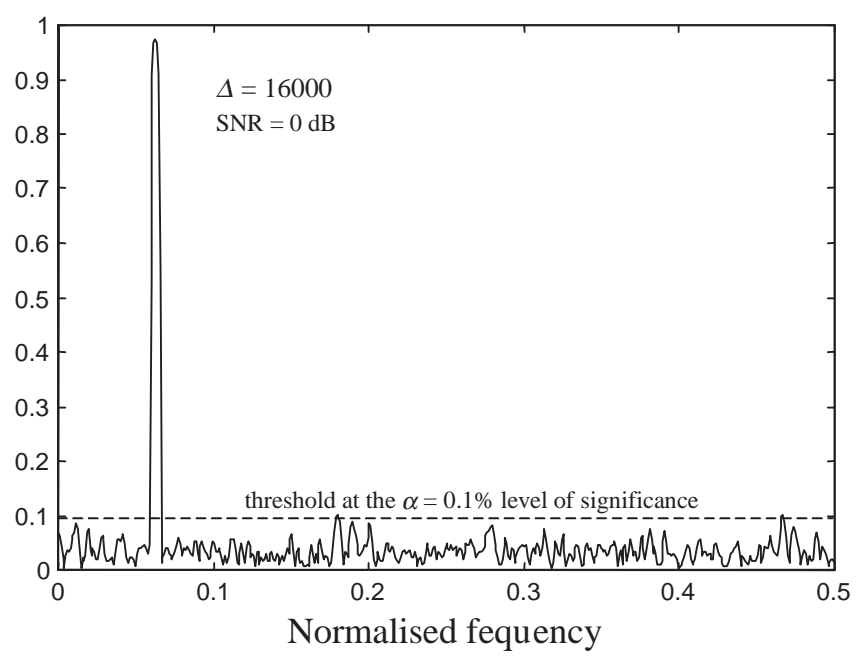

Fig. 10. Estimated indicator $\left|G^{\Delta}(f)\right|$ on the signal of Fig. 1. Signal-to-noise ratio $=0 \mathrm{~dB}$. For $\Delta>16000$, the two resonance can unambiguously be recognised as not being tones.

\section{Application to actual signals}

The application described hereafter concerns a paper machine designed and mounted some decades ago, with a specific velocity range of the paper strip. A paper machine under operation represents an interesting system to test such a kind of technique, because it is mainly built as a huge mechanical system of hundreds of different diameter cylinders, driven by a single belt, few meters large, which is the paper strip under forming. Obviously, the paper is driven by the cylinders, moved by electrical motors, but, under the kinematic point of view, the difference is negligible. Cylinders are very heavy, even tenth of tons each, and usually well balanced unless a residual unbalance is always present. The different diameters of cylinders are the origin of the different rotating speeds; each cylinder is hence a source for an harmonic excitation. The output-only analysis was devoted to predict the possible consequences over a machine section, due to the speed increase needed to increase the production. An impulsive excitation was first applied to the most critical part of the machine (with zero paper strip velocity), to detect eigenfrequencies and mode shapes of the structure. Then the machine was given the normal speed to quantify the actual excitation sources due to unbalances and to validate the modal parameter extraction of the previous step. Clearly a "coloured" excitation was present in the latter case.

Figure 11 shows the power spectral densities of some acceleration signals captured at different locations on the machine. The sampling frequency is $256 \mathrm{~Hz}$ and the frequency resolution is $0.5 \mathrm{~Hz}$. Numerous peaks are clearly noticeable in the spectra, which are indicated by vertical dotted lines. The question is which of these peaks are produced by the kinematics of the machine, and which are assigned to natural vibration modes.

Results of the CVA are shown in Fig. 12.

The spectral analysis of the vibration signals has revealed many peaks in the frequency domain. However, the kinematics of the system indicates that only a few of them can be related to the characteristic frequencies of rotating parts, i.e. the cylinders with different diameters. The other peaks are suspected to be generated by resonances. In order to validate this opinion, the techniques discussed in this paper have been tested and compared.

Figure 13(a) displays the results obtained from the FDD technique. It can be seen that the so-obtained singular spectra are difficult to interpret. Indeed, if one applies the rule that a pure tone shares its power among several singular values, then most of the peaks of Fig. 13(a) should be assigned to harmonics. This is in contradiction with the system kinematics.

Figure 13(b) displays the results obtained from the "family of spectra" technique. Here again, most if not all of the peaks are recognised as harmonics. This results supports the analysis of Section 3.6 where it was demonstrated that the "family of spectra" technique has an exaggerated tendency to classify spectral peaks as pure tones. 


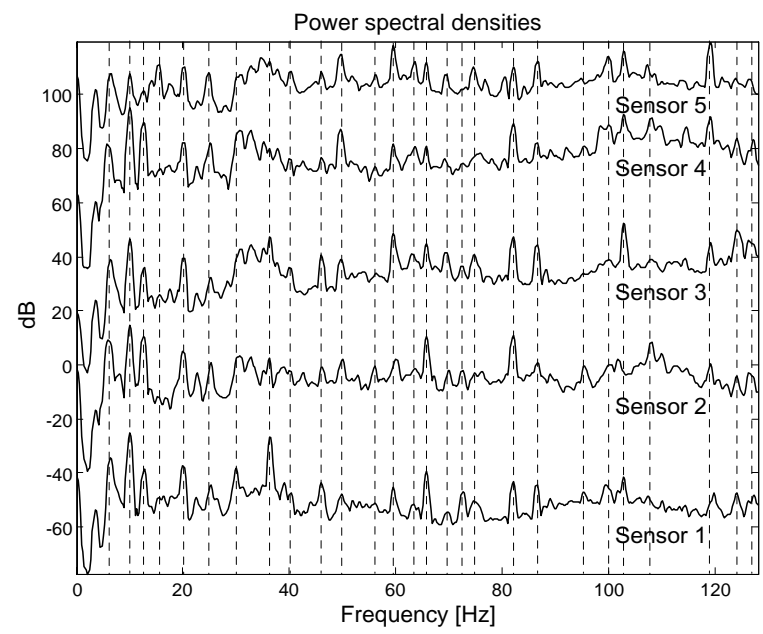

Fig. 11. Power spectral densities computed at different sensors. The spectra have been purposely shifted along the vertical axis for better visualisation. Vertical dotted lines indicate the peaks of greatest power.

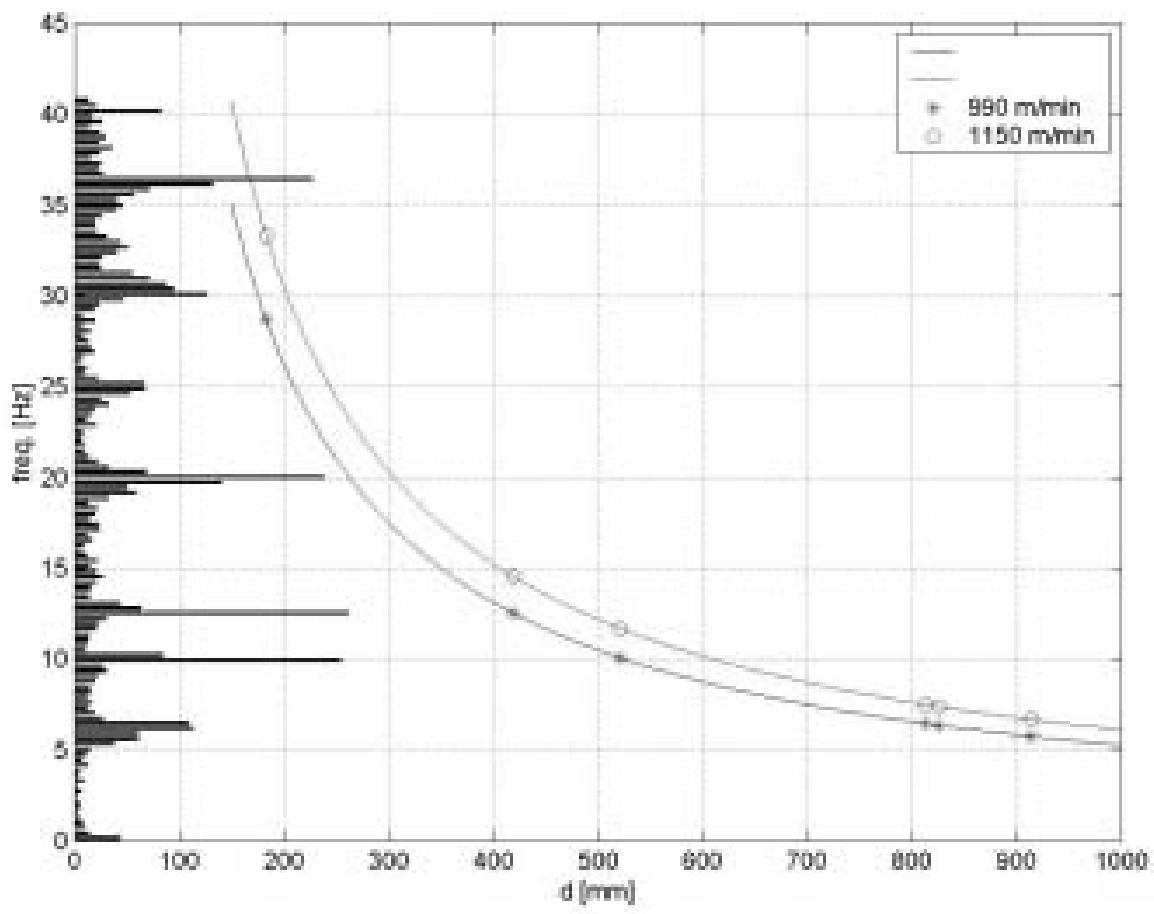

Fig. 12. CVA on the six-channel signal: on the left is shown the occurrence histogram of the extracted frequencies at the current velocity of the paper strip [990 m/1']. Asterisks and circles denote respectively the unbalances at the current velocity and at the future velocity [1150 m/1'], d indicating the diameter of the cylinders in the machine section under test.

Figure 14(a) displays the results obtained with the envelope indicator. These exhibit a clear difference with those previously obtained. A number of spectral peaks are now recognised as not being true harmonics. This is for instance the case for the major peaks at $20 \mathrm{~Hz}, 25 \mathrm{~Hz}, 49.5 \mathrm{~Hz}$, and $65.5 \mathrm{~Hz}$ which are undoubtedly related with lightly damped resonances of the system.

Finally, Fig. 14(b) displays the results obtained with the correlation time indicator. These are very similar to those of the envelope indicator, and results were found to stabilise as soon as $\Delta=1000$. The only difference is that 


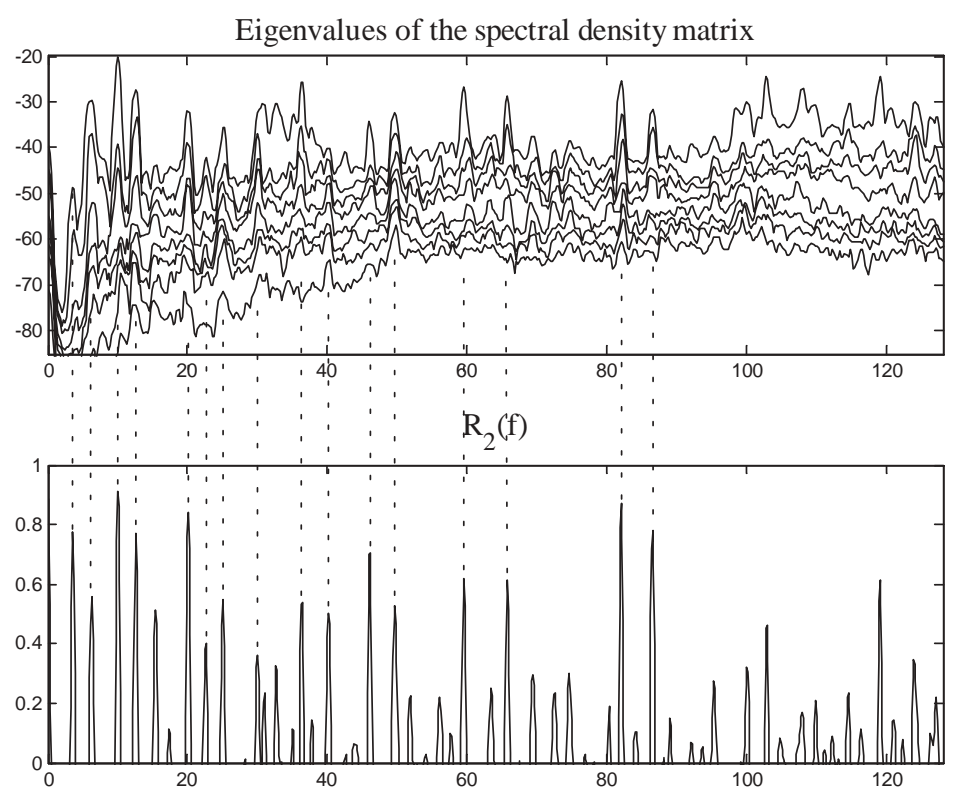

Frequency $[\mathrm{Hz}]$

Fig. 13. a) The FDD applied to the six-channel signal. This results in six singular spectra sharing the total power of the signals. b) The estimated indicator $R_{q}(f), q=2$ (average over the six channels).
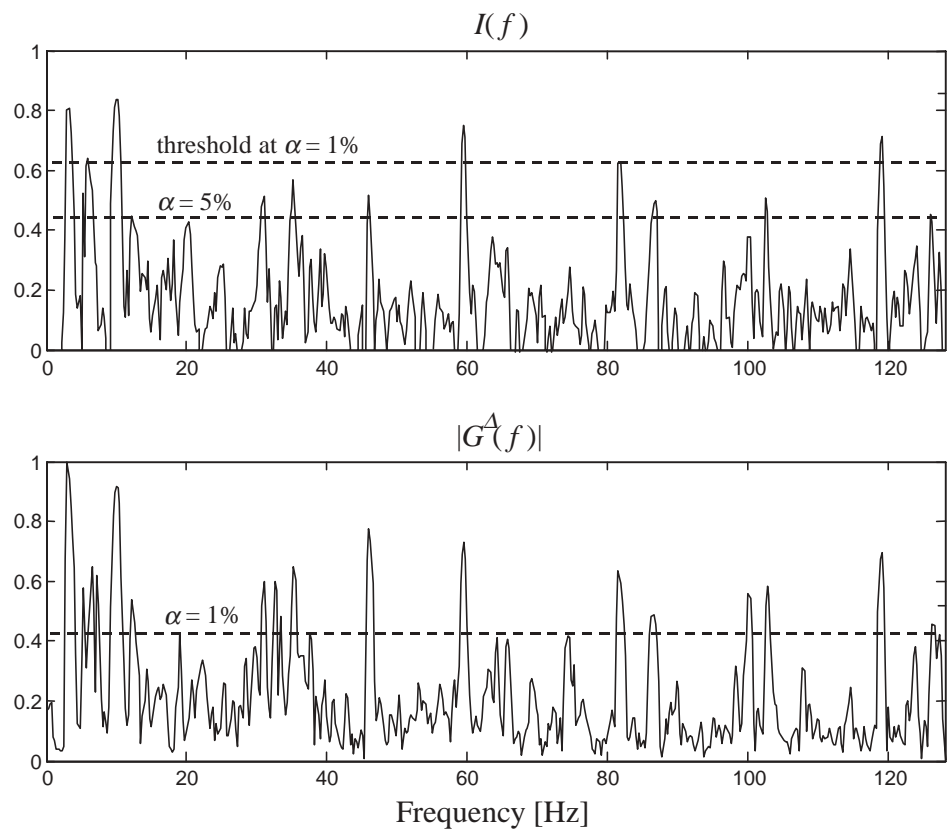

Fig. 14. a) The envelope indicator and b) the correlation time indicator with $\Delta=1000$ averaged over the 6 channels.

harmonics are recognised with a smaller level of significance - i.e. probability of type I error.

Compilations of the results from Figs 14(a) and 14(b) leads to useful observations that can now be used to perform the output-only modal analysis of the system on a sounder basis. These are summarised in Table 1. 
Table 1

Characterisation of the greatest identified peaks in the range $[0 ; 100 \mathrm{~Hz}]$

\begin{tabular}{ccc}
\hline Position & True harmonic & Resonance \\
\hline 6.5 & $\times$ & \\
10 & $\times$ & \\
12.5 & $\times$ & $\times$ \\
20 & & $\times$ \\
25 & $\times$ & \\
30 & $\times$ & \\
36.5 & & \\
40 & $\times$ & \\
46 & & \\
49.5 & $\times$ & $\times$ \\
59.5 & & $\times$ \\
63.5 & & $\times$ \\
69.5 & & $\times$ \\
65.5 & & $\times$ \\
72.5 & & \\
74.5 & $\times$ & \\
82 & & \\
95.5 & $\times$ & \\
86.5 & & \\
\hline
\end{tabular}

\section{Conclusion}

This paper was concerned with the issue of discriminating between pure tones and sharp-pointed resonances of vibrating systems. This is a critical problem that arises in many engineering applications, and especially in output-only modal analysis where it is of prime importance to differentiate operational modes from natural modes with low damping.

The issue was shown to be difficult to solve. A number of existing approaches have been reviewed, but none of them proved completely satisfying. Therefore we have proposed two new approaches, with the requirements that they should be as universal as possible and robust against additive noise.

The first approach makes use of the property that a pure tone is deterministic whereas a random noise is not. This property was found to materialise well on the complex envelope of spectral components, from which an ad hoc indicator was derived. The second approach makes use of the property that the autocorrelation function of a pure tone is periodic whereas that of a random noise is transient.

The effectiveness of our approaches was demonstrated on simulated and actual signals. The key advantages of the proposed indicators are twofold. First, they are simple and fast to compute, because they are based on FFT estimation techniques. Second, they can be complemented by statistical tests that provide answers as whether a spectral peak is a pure tone or not within given levels of significance, i.e. probabilities of error.

\section{References}

[1] M.S. Bartlett, Symposium on Spectral Approach to Time Series, J. Roy. Statist. Soc. Ser. B 19 (1957), 1-63.

[2] R.A. Fisher, Tests of Significance in Harmonic Analysis, Proc. Roy. Statist. Soc. Ser. A 125 (1929), 54-59.

[3] P. Whittle, The Simultaneous Estimation of a Time Series Harmonic Covariance Structure, Trabajos. Estadist. 3 (1952), $43-57$.

[4] E.J. Hannan, Testing for a Jump in the Spectral Function, J. Roy. Statist. Soc. Ser. B 23 (1961), 394-404.

[5] M.B. Priestley, The Analysis of Stationary Processes with Mixed Spectra - I, J. Roy. Statist. Soc. Ser. B 24 (1962), $215-233$.

[6] M.B. Priestley, The Analysis of Stationary Processes with Mixed Spectra - II, J. Roy. Statist. Soc. Ser. B 24 (1962), 511-529.

[7] V.F. Pisatrenko, The Retrieval of Harmonics from a Covari- ance Function, Geophysics J. Roy. Astron. Soc. 33 (1973), $347-366$.

[8] R. Schmidt, Multiple Emitter Location and Signal Parameter Estimation, Proc. RADC Spectrum Estimation Workshop (1979), $243-258$.

[9] M.H. Hayes, Statistical Digital Signal Processing and Modelling, New York Wiley 1996.

[10] F. Varadi et al., Searching for Signal in Noise by Random-Lag Singular Spectrum Analysis, The Astrophysical Journal 526 (1999), 1052-1061.

[11] L. Ljung, System Identification: Theory For The User, Prentice-Hall 1987.

[12] P. Van Overschee and B. De Door, Subspace identification for linear systems, Kluwer Academic Publishers 1996. 
[13] U.B. Desai, P. Debajyoti and R.D. Kirkpatrick, A realisation approach to stochastic model reduction, Int. J. of Control 42(4) (1985), $821-838$.

[14] L. Hermans and H. Van der Auweraer, Modal testing and analysis of structures under operational conditions: industrial applications, Mech Syst. and Signal Processing 13(2), 193-216.

[15] D. Spina, L. Garibaldi, S. Marchesiello, E. Giorcelli and A. Fasana, Different analysis techniques applied to seismic data from the 1997 Italian earthquake, DAMAS99 Conference, Dublin, Ireland, 28-30 June 1999.

[16] B.A.D. Piombo, L. Garibaldi, E. Giorcelli and S. Marchesiello, CVA-ARMAV capabilities comparison over the Heritage Court Tower data, Proceedings of the International Modal Analysis Conference - IMAC 2000, San Antonio (Texas), USA, 7-10 February 2000.

[17] A. Fasana, L. Garibaldi, S. Marchesiello and S. Sorrentino, An Error Estimate for CVA, DAMAS 2001 Conference, Cardiff, UK, 25-27 June, 2001.

[18] M. Prevosto, Algorithmes d'Identification des Caractéristiques Vibratoires des Structures Mécaniques Complexes, PhD Thesis, Université de Rennes I, France, 1982, in French.

[19] R. Brincker, L. Zhang and P. Andersen, Modal identification of output-only systems using frequency domain decomposition, Smart Mater. Struct. 10 (June 2001), 441-445.

[20] R. Brincker, P. Andersen and N. Møller, An Indicator For Separation Of Structural And Harmonic Modes In Output-Only Modal Testing, Proceedings of the International Modal Analysis Conference - IMAC 2000, San Antonio (Texas), USA, 7-10 February 2000, pp. 16491654.

[21] C.Y. Shih et al., Complex Modes identification Function and its Application to Spatial Domain Parameter Estimation, Mech. Syst. Signal Processing 2(4) (1988), 367-377.

[22] C. Foias, A.E. Frazho and P.J. Sherman, A Geometric Approach to the Maximum Likelihood Spectral Estimator for Sinusoids in Noise, IEEE Trans. IT 34 (Sept. 1988), 1066-1070.

[23] P.J. Sherman and K.-N. Lou, On the Family of ML Spectral Estimates for Mixed Spectrum Identification, IEEE Trans. on Signal Processing 39(3) (March 1991), 644-655.

[24] J. Antoni and R.B. Randall, Unsupervised Noise Cancellation for Vibration Signals: part II - A Novel Frequency-Domain Algorithm, Mech. Syst. Signal Processing 18(1) (January 2004), 103-117.

[25] J. Antoni and R.B. Randall, Unsupervised Noise Cancellation for Vibration Signals: part I - Evaluation of Adaptive Algorithms, Mech. Syst. Signal Processing 18(1) (January 2004), 89-101. 

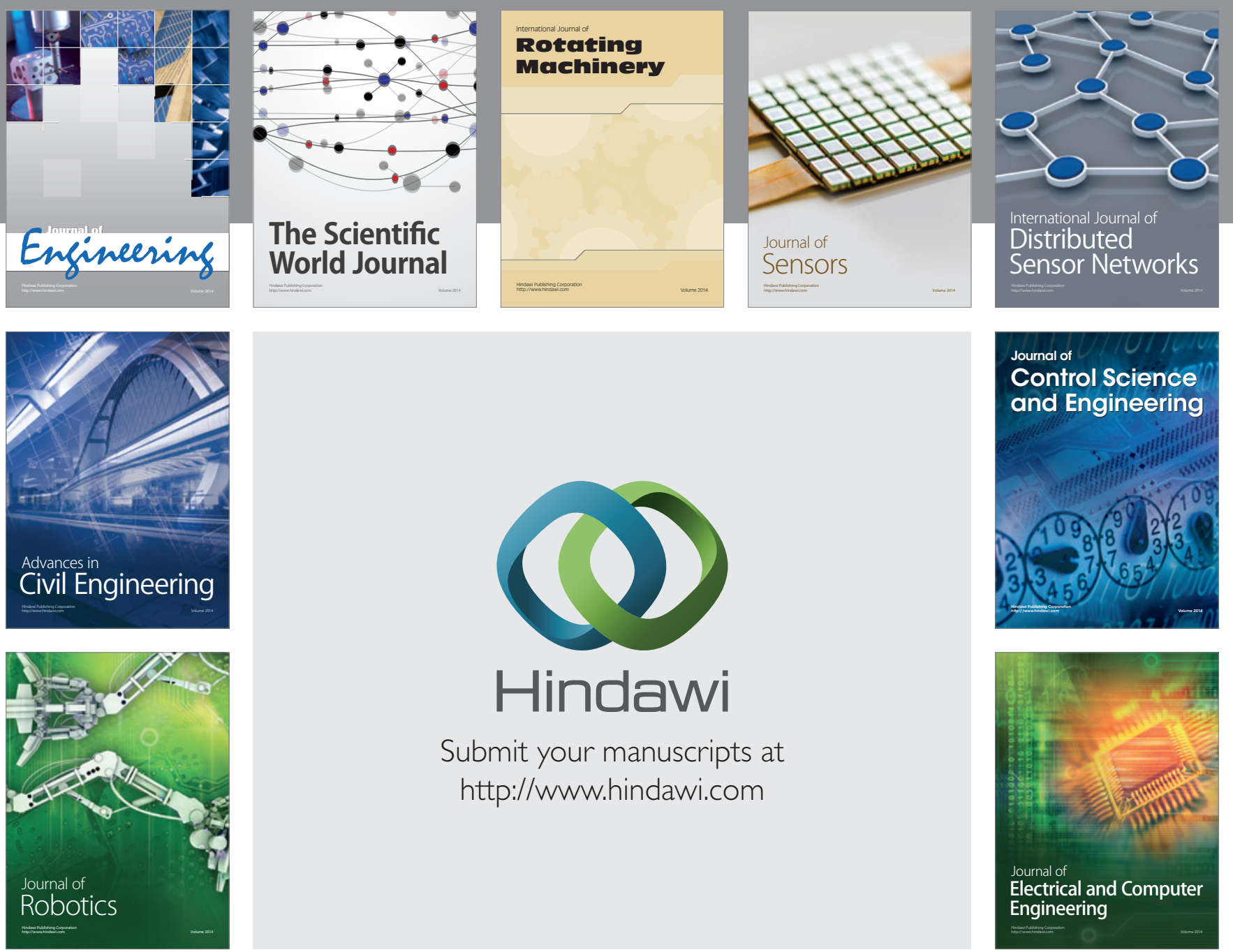

Submit your manuscripts at

http://www.hindawi.com
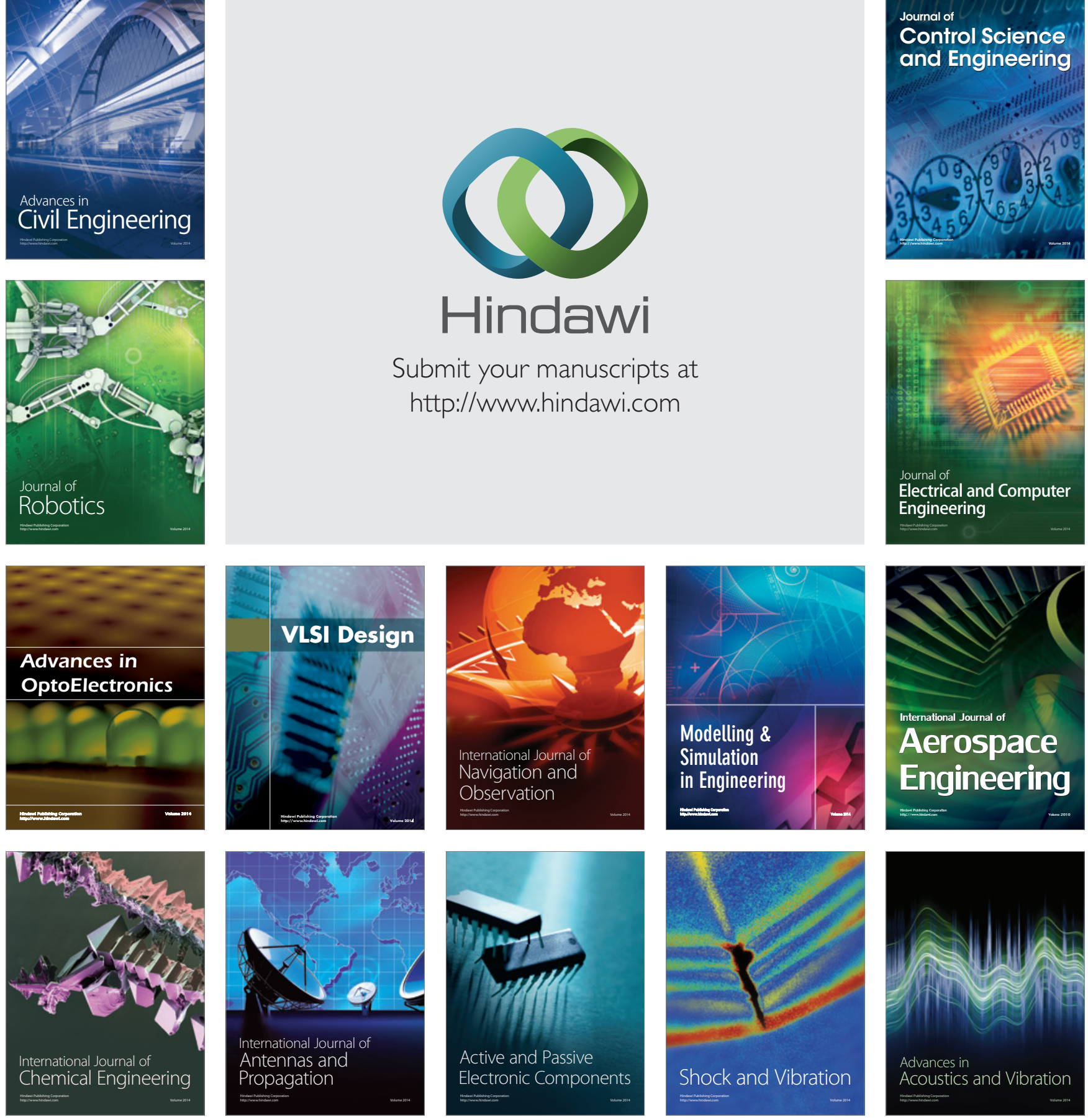